\title{
Defects in oxide surfaces studied by atomic force and scanning tunneling microscopy
}

\author{
Thomas König, Georg H. Simon, Lars Heinke, Leonid Lichtenstein \\ and Markus Heyde*
}

\author{
Review \\ Address: \\ Fritz-Haber-Institut der Max-Planck-Gesellschaft, Faradayweg 4-6, \\ 14195 Berlin, Germany \\ Email: \\ Markus Heyde* - heyde@fhi-berlin.mpg.de \\ * Corresponding author \\ Keywords: \\ aluminum oxide; charge state; contact potential; defects; domain \\ boundaries; dynamic force microscopy; frequency modulation atomic \\ force microscopy; Kelvin probe force microscopy; magnesium oxide; \\ non-contact atomic force microscopy; scanning tunneling microscopy; \\ thin films; work function
}

Beilstein J. Nanotechnol. 2011, 2, 1-14.

doi:10.3762/bjnano.2.1

Received: 01 October 2010

Accepted: 09 December 2010

Published: 03 January 2011

This article is part of the Thematic Series "Scanning probe microscopy and related methods".

Guest Editor: E. Meyer

(C) 2011 König et al; licensee Beilstein-Institut.

License and terms: see end of document.

\begin{abstract}
Surfaces of thin oxide films were investigated by means of a dual mode NC-AFM/STM. Apart from imaging the surface termination by NC-AFM with atomic resolution, point defects in magnesium oxide on $\operatorname{Ag}(001)$ and line defects in aluminum oxide on $\mathrm{NiAl}(110)$, respectively, were thoroughly studied. The contact potential was determined by Kelvin probe force microscopy (KPFM) and the electronic structure by scanning tunneling spectroscopy (STS). On magnesium oxide, different color centers, i.e., $\mathrm{F}^{0}$, $\mathrm{F}^{+}$, $\mathrm{F}^{2+}$ and divacancies, have different effects on the contact potential. These differences enabled classification and unambiguous differentiation by KPFM. True atomic resolution shows the topography at line defects in aluminum oxide. At these domain boundaries, STS and KPFM verify $\mathrm{F}^{2+}$-like centers, which have been predicted by density functional theory calculations. Thus, by determining the contact potential and the electronic structure with a spatial resolution in the nanometer range, NC-AFM and STM can be successfully applied on thin oxide films beyond imaging the topography of the surface atoms.
\end{abstract}

\section{Review}

\section{Introduction}

The chemical properties of many crystal surfaces, especially oxides, are significantly influenced by defects in the perfectly ordered structure [1-5]. These defects can be impurities in the surface, interstitials, vacancies or adsorbates. Furthermore, any deviation from the crystalline pattern constitutes such a defect [6]. These defects in the pristine surface may be generated by 
bombardment with particles, irradiation or contamination with adsorbates. Defects may also be generated during growth. For instance, defects in thin films may be caused by a lattice mismatch between film and substrate. This may result in a rather frequent and sometimes regular occurrence of the defects. Defect types can be conveniently classified by the dimensionality of their spatial extension, i.e., as point, line and planar defects. Apart from perturbations of the topography and the stoichiometry, most defects exhibit special electronic structures, which significantly differ from the pristine surface. In many cases, it is exactly this deviating electronic structure which produces various special properties of the surface. For example, defects are often preferred adsorption sites and hence are particularly chemically active. Electrically charged defects may enable electron transfer processes, which play an important role in chemical reactions in general and in heterogeneous catalysis in particular. A sketch of a binary oxide surface including several point defects is shown in Figure 1. These point defects could be color centers, where the site of a missing oxygen atom may be empty or occupied by one or more electrons.

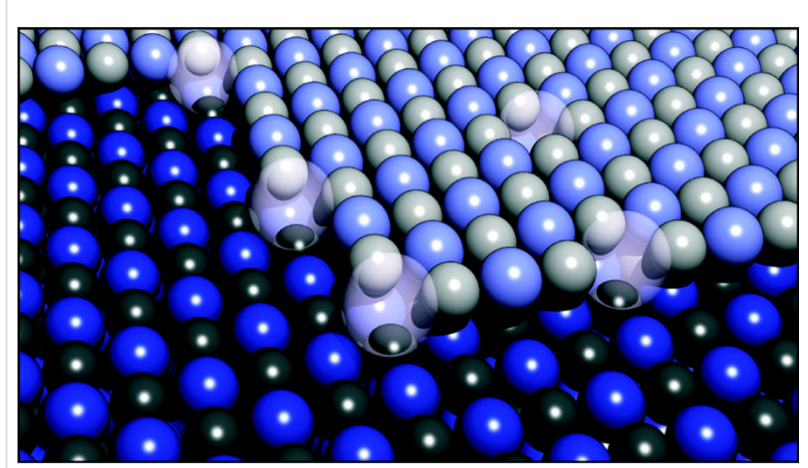

Figure 1: Model of a binary oxide surface. Point defects such as color centers, which are preferably situated at lower coordinated sites, are sketched as bright clouds.

In this publication, we review the recent work of our group, where the structure and the topography of defects in oxide surfaces was studied by non-contact atomic force microscopy (NC-AFM) and scanning tunneling microscopy (STM). Furthermore, the contact potential was determined by Kelvin probe force microscopy (KPFM). This technique has a high spatial resolution, thus avoiding averaging over various defects. Here, we confine ourselves to different point defects in magnesium oxide and to line defects in aluminum oxide. Both samples were prepared as thin films on metal supports. As a consequence, STM and scanning tunneling spectroscopy (STS) can be performed and conclusions about the electronic structure of the defects and the pristine film can be drawn. This enables a direct comparison with NC-AFM results. The application of NC-AFM and KPFM in combination with STM and STS allows a detailed investigation of the topography as well as of the contact potential and the energetic structure of the defects.

\section{Experimental setup: dual mode NC-AFM/ STM}

The employed scanning probe microscope, i.e., a NC-AFM in combination with a STM, was optimized for surface investigation on the atomic scale with spatial resolution of some picometers. Note that NC-AFM is frequently referred to as frequency modulation atomic force microscopy (FM-AFM) or dynamic force microscopy (DFM).

For the stability of tip and sample as well as for the reduction of piezo creep, piezo hysteresis, thermal drift and noise level, the setup was operated in ultrahigh vacuum (UHV) at low temperature $(5 \mathrm{~K})$. The resulting high stability makes atomic resolution on conductors [7] as well as on insulators [8] possible. In addition to investigations on the surface topography, site specific spectroscopy measurements can be performed [8]. The whole setup is placed in a sound absorber cabin and is carried on a wooden frame, which, in turn, is based on an active vibrational damping system. The background pressure in the UHV chamber is below $4 \times 10^{-8} \mathrm{~Pa}$. The microscope stage is cooled down with a liquid helium bath cryostat (Figure 2a). A so-called exchange gas canister is situated between microscope compartment and helium bath. The exchange gas canister is filled with helium gas to a pressure of about $1000 \mathrm{~Pa}$. The helium gas establishes thermal coupling between the microscope stage inside the UHV chamber and the liquid helium inside the bath cryostat. In addition, the vibrations caused by the evaporating helium inside the bath cryostat are decoupled from the microscope.

The dual mode NC-AFM/STM sensor (Figure 2c) is situated on a tripod scanner opposite the sample. The scanner, in turn, is mounted onto a coarse approach unit (walker). The microscope stage is shown in Figure 2 b. The coarse approach is driven by the shear stack piezos. If the tip-sample distance reaches the range of interatomic forces or the tunneling regime, the walker is switched off and the scan is performed by the $x, y$ and $z$ piezos. An additional excitation piezo orientated along $z$ excites the tuning fork at resonance. The tuning fork sensor is presented in Figure 2c. The tuning forks were made of quartz $\left(\mathrm{SiO}_{2}\right)$ and are, therefore, piezo electric devices. Because of their very stable oscillation properties upon electric excitation, they are widely used in watches. Commercial tuning forks have often a resonance frequency of $32768 \mathrm{~Hz}\left(=2^{15} \mathrm{~Hz}\right)$. In the employed setup, one prong of the tuning fork is glued onto the carrier. A $\mathrm{Pt}_{0.9} \mathrm{Ir}_{0.1}$ wire, $250 \mu \mathrm{m}$ in diameter, is attached to the other prong as a tip. The use of a non-conducting glue electrically 
a)

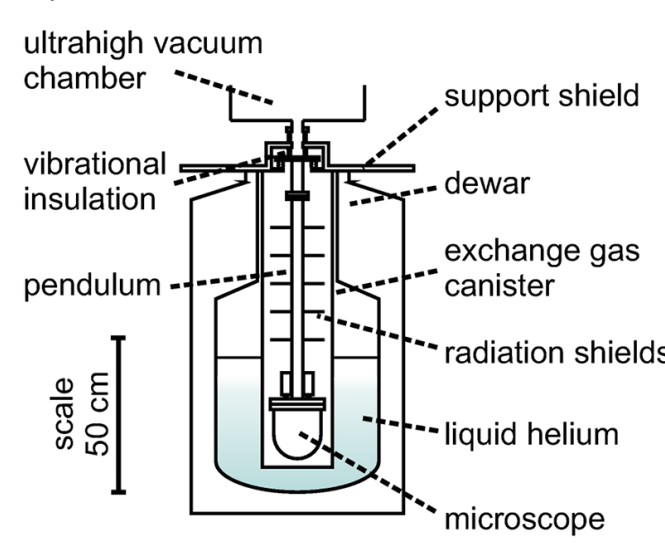

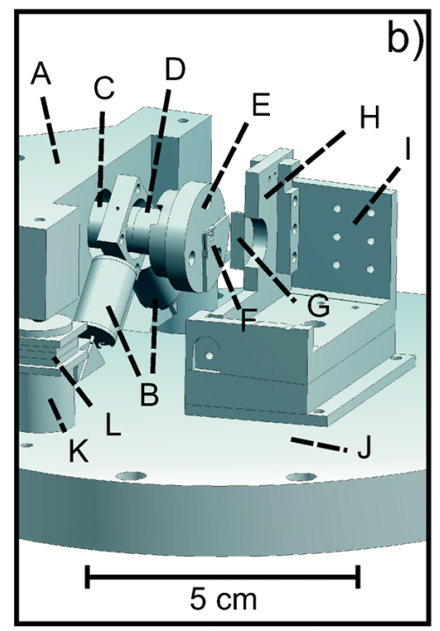

b)

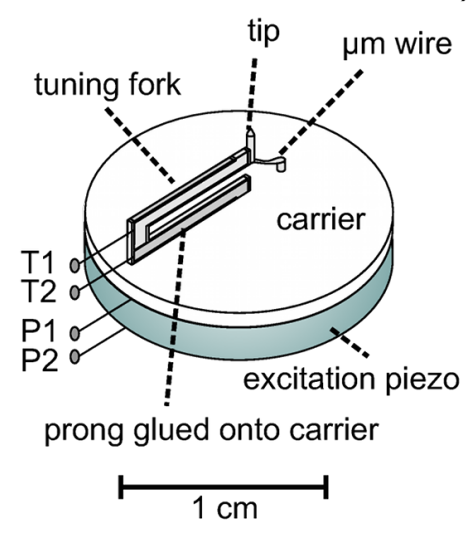

Figure 2: Experimental setup. a) Schematic of an Eigler-style bath cryostat. b) The walker unit is situated on three shear stack piezos for the coarse approach. The $x, y$ and $z$ piezos are used during the scan process. The tuning fork is located opposite the sample (only half of the sample is drawn to keep the view free to the sensor carrier). Schematic of the microscope on its support stage: (A) walker unit, (B) $x, y$ piezo and (C) $z$ piezo of the tripod scanner unit, (D) $z$ dither piezo, (E) sensor carrier, (F) tuning fork assembly, (G) sample (not fully drawn), (H) sample holder (not fully drawn), (I) sample stage (not fully drawn), (J) microscope stage, (K) walker support and $(\mathrm{L})$ shear stack piezos. The base plate has a diameter of $10 \mathrm{~cm}$. $\mathrm{C}$ ) The NC-AFM/STM tuning fork sensor is glued onto the carrier made of MACOR. Contacts P1 and P2 are the contacts of the excitation piezo. The signal from the tuning fork is detected via contact $\mathrm{T} 1$ and $\mathrm{T} 2$. The $\mu \mathrm{m}$ wire attached to the tip conducts the tunneling current.

insulates the tip from the tuning fork and prevents cross talk. Due to the fixed prong and the additional mass of the tip at the other prong, the resonance frequency drops to about $22 \mathrm{kHz}$.

The tuning fork is driven by the excitation piezo. Due to the piezo electric effect, the signal of the resonance frequency can be detected at the electrodes of the tuning fork. The amplitude of the signal is proportional to the oscillation amplitude of the tuning fork. The signal is so small that a low-temperature amplifier has to be placed nearby to improve the signal-to-noise ratio. In NC-AFM, the shift of the tuning fork resonance frequency $\Delta f$ is used as a feedback signal to scan with constant $\Delta f$. The tip is electrically connected to a wire, $50 \mu \mathrm{m}$ in diameter (see Figure 2c). Using this electrical contact, a bias voltage can be applied between tip and sample and a tunneling current $I_{\mathrm{t}}$ can be measured. $I_{\mathrm{t}}$ serves as a feedback signal when operating in the STM mode at constant current. While operating in one of the modes, NC-AFM or STM, the other channel can always be co-recorded. Great care was taken to ensure that both channels, NC-AFM and STM, were electrically separated from each other in order to prevent cross talk.

The great advantage of this setup is the simultaneous data acquisition of the frequency shift and the tunneling current, making it a powerful tool for high resolution real space analysis at the atomic level and merging the strengths of both techniques. The combination of both techniques enables the detection of contaminants on the tip. For instance, insulating contam- inants cause a shift of the minimum of the $\Delta f$ signal to larger tip-sample distances, whereas $I_{\mathrm{t}}$ is not influenced. In general, it is interesting to measure both signals as they complement each other and the use of the very same microscopic tip enables direct comparison. Pairs of curves from both channels recorded in a sweep in $z$ direction and another one recorded at varying bias voltage are shown in Figure 3.

\section{Spectroscopic methods: tip-sample forces in NC-AFM}

In surface science, forces detectable by NC-AFM in UHV at low temperature have been classified into three main categories [9]. The first category has an electrostatic origin and covers forces between charges, also known as Coulomb forces. These forces arise from the interaction between charges, permanent dipoles and higher order moments. Polarization forces are the second category. These forces cause dipole moments in atoms or molecules, which are induced by electric fields of charges and of permanent or induced dipoles. The third category covers bonding forces, which have a quantum mechanical nature. These forces lead to charge transfer processes as involved in covalent bonding. Furthermore, this category includes the repulsive exchange forces, which are caused by the Pauli exclusion principle. These repulsive forces balance and prevail the attractive forces at very short distances. The classification into these three groups is neither rigid nor exhaustive. For example, van der Waals force, which falls into category two, is a general consequence of the zero-point energy in quantum mechanics 

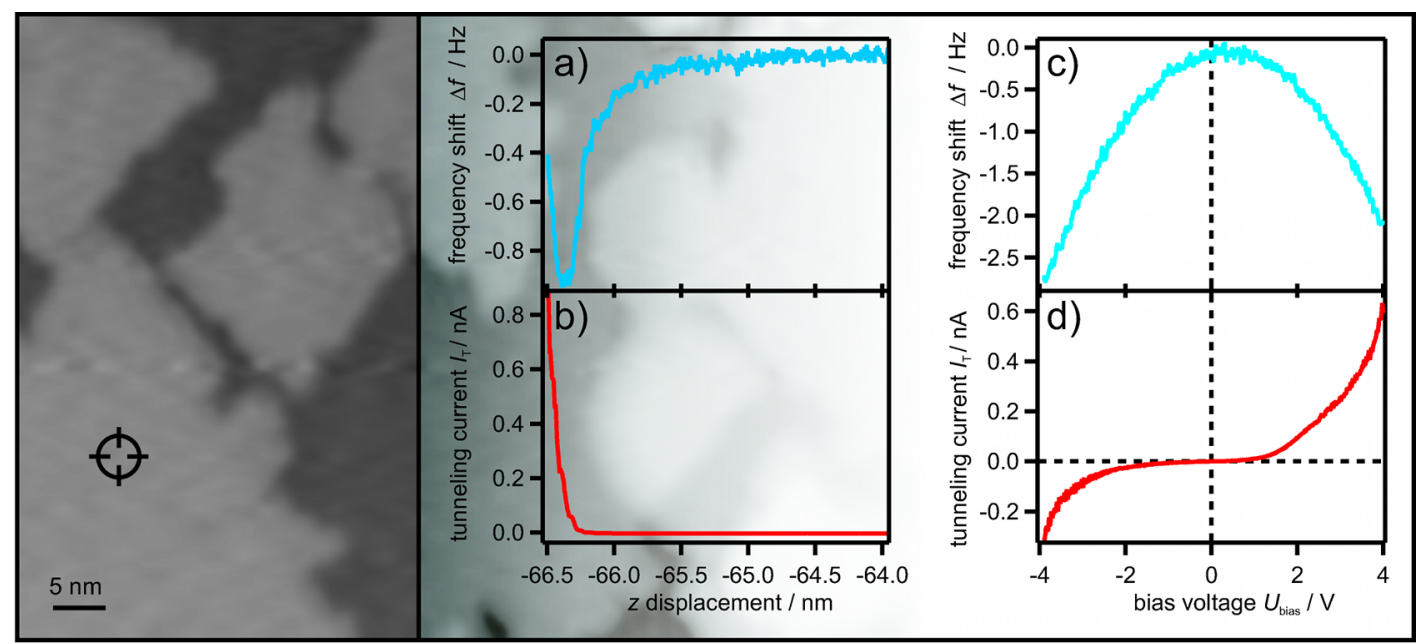

Figure 3: The same tip senses both signals. (a-d) Pairs of simultaneously recorded signal curves from the frequency shift and the current channel: $(a, b)$ signal-distance curves at constant bias voltage, (c,d) signal-bias voltage curves at constant height. On the left-hand side, a STM image of the $\mathrm{MgO}$ film recorded with a bias voltage of $+3.5 \mathrm{~V}$ and a tunneling current of $100 \mathrm{pA}$ is shown. The tip position for the spectroscopy is indicated.

$[10,11]$. Furthermore magnetic forces, friction forces, capillary forces etc. can in principle occur in NC-AFM. These forces are not relevant in this paper, since, e.g., a magnetic tip is necessary to detect magnetic forces, or non conservative forces have to be measured to determine friction forces.

The forces relevant in this work are described below. Coulomb forces are a result of interacting charges and can be stronger than most chemical binding forces [9]. The Coulomb potential $E_{\text {Coulomb }}$ between two charges $Q_{1}$ and $Q_{2}$ is given by

$$
E_{\text {Coulomb }}(z)=\frac{Q_{1} Q_{2}}{4 \pi \varepsilon_{0} \zeta z},
$$

where $\varepsilon_{0}$ is the permittivity constant, $\zeta$ is the relative permittivity or dielectric constant of the medium and $z$ the distance between the charges. The Coulomb force $F_{\text {Coulomb }}$ is given by

$$
F_{\text {Coulomb }}=-\frac{d E_{\text {Coulomb }}}{d z}=\frac{Q_{1} Q_{2}}{4 \pi \varepsilon_{0} \zeta z^{2}} .
$$

It is well known [12] that for very small amplitudes, the shift of the resonance frequency $\Delta f$ corresponds to the derivative of the tip-sample forces with respect to $z$. For larger amplitudes, a more general relation can be derived [12], which is not always proportional, however, strictly monotonic. Consequently, the tip-sample forces and potentials can be determined by recording $\Delta f$ with NC-AFM.

Via detection of electrostatic forces, contact potentials can be determined by NC-AFM in the KPFM mode [13-16], which is named after Lord Kelvin, who measured contact potentials in a similar way [17]. The contact potential (CP) results from the alignment of the Fermi levels of tip and sample having different work functions. The tip-sample geometry can be considered as a capacitor, resulting in the following equation for the electrostatic energy $\mathrm{E}_{\mathrm{el}}$, which together with the non-electrostatic interaction such as a Lennard-Jones potential adds to the total energy, $[18,19]$

$$
\begin{aligned}
E_{\mathrm{el}} & =E_{\mathrm{charge}}-E_{\mathrm{vs}} \\
& =\frac{n^{2} e^{2}}{2 C_{\Sigma}}+\frac{C_{1} C_{2} U^{2}}{2 C_{\Sigma}}-\left[n e \frac{C_{1}}{C_{\Sigma}} U+\frac{C_{1} C_{2} U^{2}}{C_{\Sigma}}+\frac{C_{0}}{2} U^{2}\right] \\
& =\frac{n^{2} e^{2}}{2 C_{\Sigma}}-n e \frac{C_{1}}{C_{\Sigma}} U-\frac{C_{1} C_{2} U^{2}}{2 C_{\Sigma}}-\frac{C_{0}}{2} U^{2} .
\end{aligned}
$$

$E_{\text {charge }}$ is the energy due to electrostatic charging and $E_{\mathrm{VS}}$ is the work done by the voltage source. Furthermore, $C_{\Sigma}(z)=C_{1}(z)+$ $C_{2}$, with $C_{1}(z)$ is the capacity between the tip and a defect on the surface, $C_{2}$ is the capacity between the defect and the substrate and $C_{0}$ is the capacity between the tuning fork back electrodes and the surface. The voltage between tip and sample is given by

$$
U=U_{\text {bias }}-\frac{\Delta \Phi_{\text {loc }}}{|e|} .
$$

$U_{\text {bias }}$ is the voltage applied between tip and sample, $e$ the elementary charge, $\Delta \Phi_{\text {loc }}$ the local contact potential and $n$ represents the number of charges $e$. The derivative of Equation 3 with respect to $z$ results in the electrostatic force given by 


$$
F_{\mathrm{el}}=\frac{1}{2} \frac{\partial C_{\mathrm{S}}}{\partial z}\left(\frac{n e}{C_{2}}+U\right)^{2}+\frac{1}{2} \frac{\partial C_{0}}{\partial z} U^{2}
$$

where $C_{\mathrm{S}}$ is given by a capacitor series $C_{\mathrm{S}}=C_{1} \cdot C_{2} /\left(C_{1}+C_{2}\right)$. The last term in Equation 4 can be neglected when high resolution is considered, since the electrostatic force between the substrate and the tuning fork's back electrodes integrates a large surface area [18].

In a thin oxide film on a metal support, the surface may contain charges. It is reasonable to introduce an effective contact potential $\Delta \Phi_{\text {eff }}[20]$ which considers the shift of the contact potential of the pristine materials due to the charges in the surface, i.e., $\Delta \Phi_{\text {eff }}=\Delta \Phi-n e^{2} / C_{2}$. This results in

$$
F_{\mathrm{el}}=\frac{1}{2} \frac{\partial C_{\mathrm{S}}}{\partial z}\left(U_{\mathrm{bias}}-\frac{\Delta \Phi_{\mathrm{eff}}}{|e|}\right)^{2}
$$

Tip and sample are not directly in contact but they are electrically connected via the electronics. The electrical contact leads to an alignment of the Fermi levels of tip and sample. In Figure 4a tip and sample are not electrically connected, thus, the vacuum levels are equal and the Fermi levels do not align. In Figure $4 \mathrm{~b}$ tip and sample are electrically connected and electrons from the material with the lower work function (here tip) flow to the material with the higher work function. The Fermi levels align and an electrical field is built up [21]. The contact potential $\Delta \Phi$ is then given by the difference in work function of the tip and of the sample surface, which may contain the studied defects. By applying a bias voltage and thus reversing the charge transfer between tip and sample, the effective contact potential can be obtained as the point of minimal force (see Figure 4c). The advantage of KPFM compared with, e.g., photoelectron spectroscopy is the high local resolution down to single point defects or single adsorbates, instead of integrating over a square millimeter range. However, absolute values of the work function cannot be measured directly, only work function differences.

\section{Point defects}

Oxygen vacancies, also known as color centers, are electron trapping point defects and are supposed to be involved in electron transfer processes on the surface. The trapped electrons in the color centers can be transferred to adsorbates such as $\mathrm{Au}$ atoms. The defect-free $\mathrm{MgO}$ surface is quite inert while a defect rich surface shows a high and complex chemical reactivity [22]. In order to understand possible reaction pathways, a detailed characterization of color centers is highly desirable. Information about their local position and thus coordination, electronic structure, local contact potential and possible adsorbate interaction are of fundamental interest. In the following, color centers on the $\mathrm{MgO}$ surface are investigated in detail and classified by their charge state. From calculations it has been proposed that color centers are directly involved in chemical reactions [23,24], e.g., as adsorption sites due to more attractive defect-adsorbate interactions compared with the pristine $\mathrm{MgO}$ surface. It is also experimentally investigated whether color centers are attractive or repulsive in comparison to the surrounding $\mathrm{MgO}$ lattice.

\section{Sample system: magnesium oxide on $\mathrm{Ag}(001)$}

An NC-AFM image of a perfect $\mathrm{MgO}$ surface is shown in Figure 5. The film is two atomic layers thick, however, films with a thickness of two to eight layers give very similar images. a)

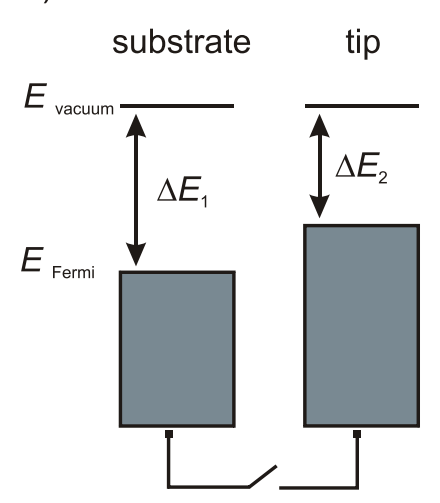

b)

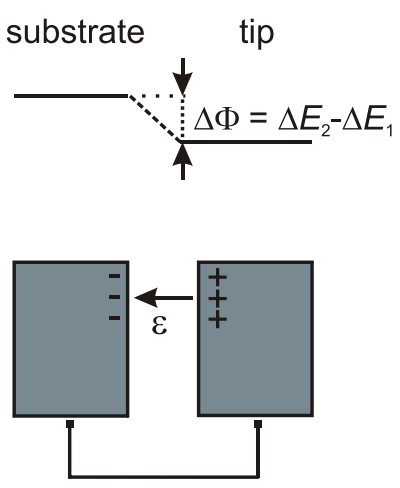

c)

substrate tip

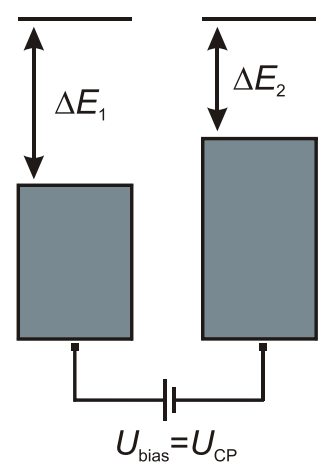

Figure 4: Energetic levels. a) The Fermi levels of tip and sample when they are not electrically connected. b) Tip and sample are electrically connected and the Fermi levels align resulting in an electrostatic field. c) If the sample bias voltage corresponds the contact potential, i.e., $U_{\mathrm{CP}}=\Delta \Phi / e$, the electrostatic field is canceled. 
One type of ion is shown as a protrusion while the other type of ion is depicted as a depression. This is a typical finding for ionic surfaces imaged by NC-AFM [25,26]. Since the density of electrons on the $\mathrm{MgO}$ surface is the highest above the oxygen atoms [27], the maxima in the NC-AFM image are thought to correspond to the positions of the oxygen atoms. Furthermore, electron paramagnetic resonance (EPR) spectra have shown that the preferred adsorption sites for $\mathrm{Au}$ atoms are on top of the oxygen ions on the terrace of the $\mathrm{MgO}$ surface [26]. Assuming that the forces acting on such metal adatoms are comparable to those on the tip apex, one may conclude that a more attractive interaction occurs between the oxygen sites and the tip. This results in a contrast where oxygen atoms are imaged as protrusions in a constant $\Delta f$ NC-AFM image.
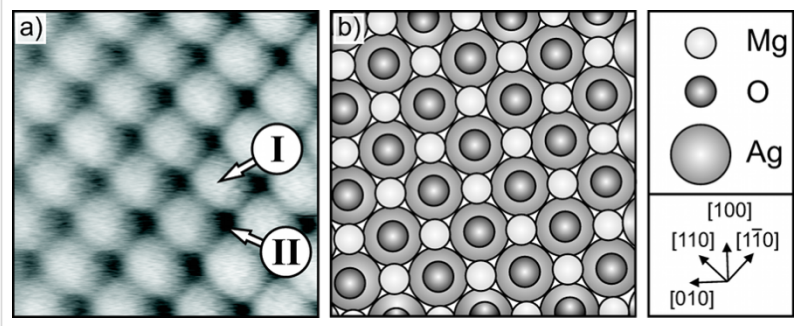

Figure 5: Magnesium oxide surface. a) Atomically resolved image recorded by NC-AFM. The position I and II indicate the two different surface atoms, oxygen and magnesium. The size is $1.5 \mathrm{~nm} \times 1.5 \mathrm{~nm}$ and the corrugation approximately $30 \mathrm{pm} . \Delta f=-8.5 \mathrm{~Hz}, A_{\mathrm{osc}}=$ $0.35 \mathrm{~nm}$. b) Schematic growth model of the $\mathrm{MgO}$ on $\mathrm{Ag}(001)$. The oxygen atoms occupy top sites, while the magnesium atoms occupy hollow sites [8].

The preparation conditions of the $\mathrm{MgO}$ film on $\mathrm{Ag}(001)$ follow a route described in [28], where a stoichiometric composition was observed. This procedure has proven its applicability in many successful preparations. The $\operatorname{Ag}(001)$ was sputtered with $\mathrm{Ar}^{+}$ions at a current density of $10 \mu \mathrm{A} / \mathrm{cm}^{2}$ and an acceleration voltage of $800 \mathrm{~V}$ for $15 \mathrm{~min}$. Afterwards, the $\mathrm{Ag}(001)$ was annealed at $690 \mathrm{~K}$ for $30 \mathrm{~min}$. The sputtering and annealing cycle was repeated several times. $\mathrm{Mg}$ was evaporated from a Knudsen cell in an oxygen atmosphere of $1 \times 10^{-4} \mathrm{~Pa}$ at a substrate temperature of $560 \mathrm{~K}$ and a deposition rate of about $1 \mathrm{ML}$ of $\mathrm{MgO} / \mathrm{min}$. A certain amount of $\mathrm{MgO}$ can be grown onto the $\mathrm{Ag}(001)$ by linear extrapolation of a sub-monolayer coverage to the desired number of monolayers, assuming a constant sticking coefficient. This preparation method is only possible since the reaction kinetics of Ag with oxygen is very slow [29] compared with the reaction between $\mathrm{Mg}$ and $\mathrm{O}$. Since the intrinsic defect density of the film is very small, color centers, such as $\mathrm{F}^{0}, \mathrm{~F}^{+}$ and $\mathrm{F}^{2+}$, have been generated by operating the microscope in the STM mode at high currents $I_{\mathrm{t}}=6 \mathrm{nA}$ and high voltages $U_{\text {bias }}=7 \mathrm{~V}$ or higher. Clean and well grown $\mathrm{MgO}$ areas have been selected to ensure defined conditions. The defects are preferentially located at kinks, corners and step edges (for an illustration see Figure 1). This means defect sites with a lower coordination number are preferred. An NC-AFM image of an $\mathrm{MgO}$ step edge with point defects is shown in Figure 6.

\section{Color centers in magnesium oxide}

The high local resolution of the NC-AFM image shown in Figure 5 serves as the starting point for adsorbate-defect interaction studies. The tip, representing the adsorbate, scans laterally across the defect positions at constant height along the step direction. The simultaneously measured frequency shift $\Delta f$ and tunneling current $I_{\mathrm{t}}$ give insight into the local surface potential as well as into the local electronic structure. The corresponding results of such an experiment are shown in Figure 7, where the
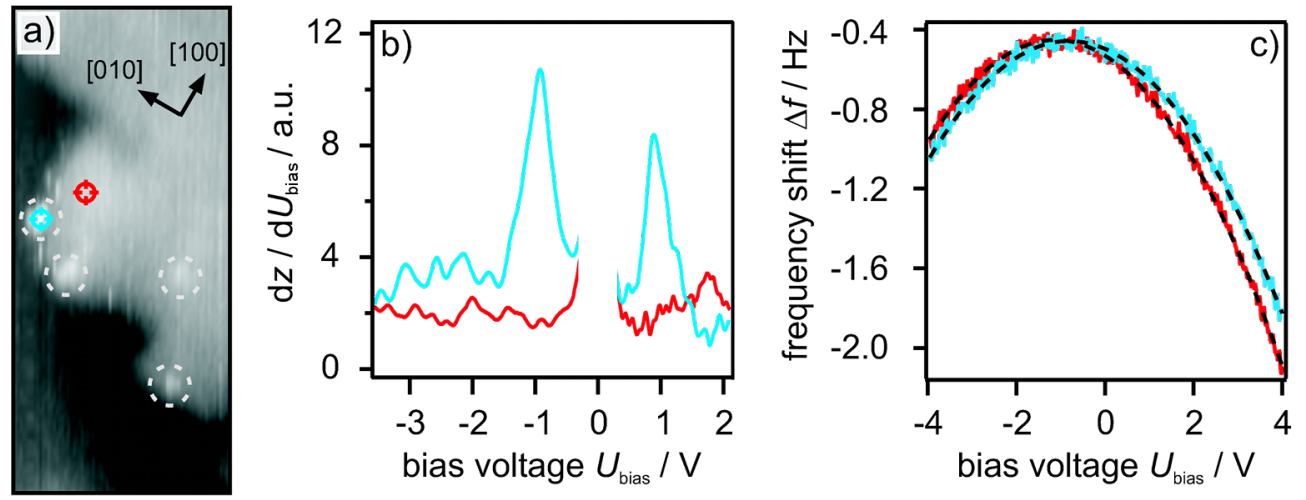

Figure 6: Spectroscopy on point defects. a) NC-AFM image of $21 \mathrm{~nm} \times 9 \mathrm{~nm}$ measured at a frequency shift of $\Delta f=-1.6 \mathrm{~Hz}$, an oscillation amplitude of $A_{\mathrm{osc}}=0.34 \mathrm{~nm}$ and $U_{\text {bias }}=-50 \mathrm{mV}$. Defects are indicated by circles. The position of the spectroscopy in b) and c) is indicated red and blue. b) STS on $\mathrm{MgO}$. There are no states in the MgO-film (red), whereas electronic defect states (blue) at approximately $+1 \mathrm{~V}$ and $-1 \mathrm{~V}$ exist. c) Frequency shift vs bias voltage spectroscopy shows a quadratic dependence at the MgO-film (red) and at the defects (blue). The maxima have different bias voltages. 

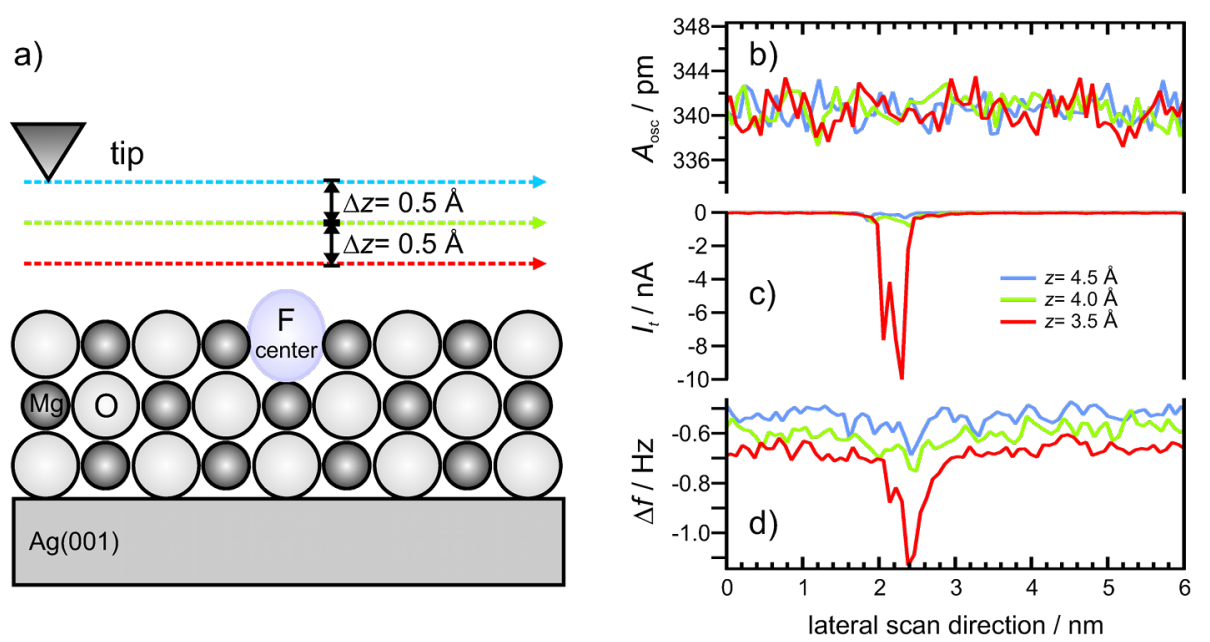

Figure 7: Dependence on tip-sample distance. Constant height line-scans across an $\mathrm{F}^{0}$ defect situated at a step edge. The scan direction is along the step edge. The three presented channels have been measured simultaneously. The colors indicate different tip-sample distances. Note that the displacement of $4.5 \AA$ has been chosen arbitrarily, since absolute values are generally unknown in scanning probe microscopy. b) The oscillation amplitude is constant during scan process. This excludes artefacts in frequency shift. c) The tunneling current and d) the frequency shift. Data were obtained at a bias voltage of $U_{\text {bias }}=-50 \mathrm{mV}$.

tip scanned across an $\mathrm{F}^{0}$ defect. The three stacked graphs show the simultaneously recorded oscillation amplitude, the frequency shift and the tunneling current. The colored traces indicate constant height scans at different tip-sample separations. At all tip-sample distances the oscillation amplitude can be considered as constant, which is a prerequisite, since the frequency shift scales with the amplitude [12].

Due to the exponential dependence of the tunneling current on the tip-sample distance, $I_{\mathrm{t}}$ vanishes at the largest separation and the shift of the resonance frequency is a consequence of the long range force background arising from electrostatic and van der Waals forces. The averaged frequency shift at the largest separation is about $\Delta f=-0.52 \mathrm{~Hz}$. By decreasing the tip-sample distance by $0.5 \AA$, the absolute value of the tunneling current and the frequency shift increase at the position of the defect. The tunneling current increases to $I_{\mathrm{t}}=-0.5 \mathrm{nA}$ and the frequency shift to $\Delta f=-0.75 \mathrm{~Hz}$ above the defect. Decreasing the tip-sample separation by another $0.5 \AA$ results in a tunneling current of $I_{\mathrm{t}}=-9.9 \mathrm{nA}$ and a frequency shift of $\Delta f=-1.13 \mathrm{~Hz}$ at the defect site. Despite the decrease of $1.0 \AA$ in tip-sample distance, the average tunneling current on the regular $\mathrm{MgO}$ terrace remains below $I_{\mathrm{t}}=-0.05 \mathrm{nA}$. The frequency shift changes by $0.15 \mathrm{~Hz}$ with decreasing tip-sample distance. This experiment demonstrates the highly attractive interaction of the tip (or adsorbate) with an $\mathrm{F}^{0}$ center.

It has been debated in literature how color centers are imaged by NC-AFM $[25,30]$ since a color center is a hole in the $\mathrm{MgO}$ lattice [22]. The observed attraction of $\mathrm{F}^{0}$ centers originates from the charge density of the two trapped electrons, which are located in the center of the defect site. Due to Coulomb repulsion, the trapped electrons repel each other and spill out of the defect site into the vacuum [31]. Therefore, a considerably large charge density is situated above the surface. This charge density is supposed to interact with the tip resulting in a strong attraction, as presented in Figure 7. Since the doubly occupied $\mathrm{F}^{0}$ state is close to the Fermi level of the $\mathrm{MgO} / \mathrm{Ag}(001)$ system [32], the charge density is also responsible for the strong peak in the tunneling current signal. Further insights into the interaction of tip and color center are obtained by periodic supercell DFT calculations at the level of the generalized gradient approximation as implemeted in the VASP code, which have been performed in the group of G. Pacchioni [33-35]. The $\mathrm{Pt}_{0.9} \mathrm{Ir}_{0.1}$ tip has been modeled by a tetrahedral $\mathrm{Pt}_{4}$ cluster, whose geometry has been relaxed separately. The $\mathrm{F}^{0}$ color center has been created by removing an $\mathrm{O}$ atom from the top layer of a three layer $\mathrm{MgO}$ slab. The structure of the slab with the color center has been relaxed. The tip-surface interaction energy has been computed as a function of tip-sample distance of the apical $\mathrm{Pt}_{4}$ cluster with respect to the top layer of the $\mathrm{MgO}$ slab (see Figure 8d). During these calculations the separately optimized tip structure was not allowed to relax. However, the relaxation of the $\mathrm{MgO}$ surface has been found to be very small for the calculated distances, where no direct contact is established. The outward relaxation of the $\mathrm{O}$ anion at $3.5 \AA$ is about $0.12 \AA$.

The results of the experimental distance dependent measurements and the corresponding theoretical results are presented in 

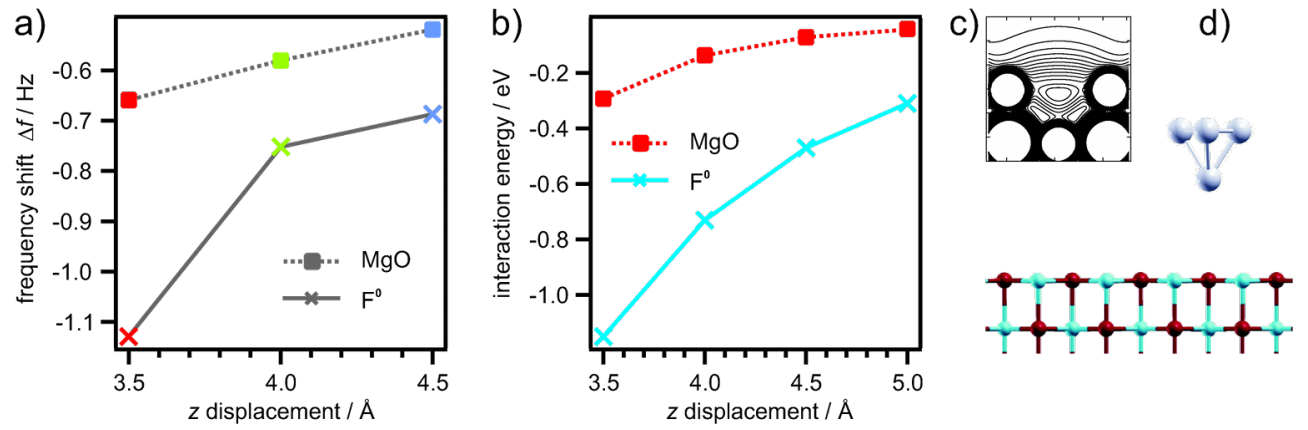

Figure 8: Dependence on tip-sample distance. a) Shift of the resonance frequency of a $\mathrm{Pt}_{0.9} \mathrm{Ir}_{0.1}$ tip on a regular MgO surface (squares) and above an $\mathrm{F}^{0}$ defect site (crosses). Experimental data are derived from the constant height measurements shown in Figure 7 . The frequency shift is a direct consequence resulting from potential gradients between tip and sample. The integration of the frequency shift is related to the potential energy. b) Interaction energy of $\mathrm{a} \mathrm{Pt}_{4}$ cluster above the $\mathrm{O}$ site of an $\mathrm{MgO}$ surface (rectangles) and above an $\mathrm{F}^{0}$ defect center (crosses) calculated by DFT. c) The spill over of the electron charge density of an $\mathrm{F}^{0}$ center calculated by DFT. d) The $\mathrm{Pt}_{4}$ cluster above the MgO surface $[35,36]$.

Figure 8. At the defect site, the tip-sample interaction increases significantly with decreasing distance. From a structural point of view the positions of the defects are "holes", i.e., missing oxygen atoms in the lattice. In the first place it is unknown which type of color center, $\mathrm{F}^{0}, \mathrm{~F}^{+}$or $\mathrm{F}^{2+}$, is imaged on the $\mathrm{MgO}$ surface. To gain further insight into the nature of the color centers we performed high resolution KPFM measurements with single point defect resolution (Figure 6). To acquire $\Delta f$ vs $U_{\text {bias }}$ curves on top of a defect, the $\Delta f$ feedback was switched off. Subsequently the frequency shift vs applied bias voltage was plotted and compared to equivalent reference measurements at the same height close to the defect. The parabolic behavior of the frequency shift curves has been analyzed with Equation 5. The electrostatic force is always attractive. This results in the parabolic dependence of the forces (see Equation 5). The maximum of the parabola depends on the local effective contact potential $\Delta \Phi_{\text {eff. It }}$ has been found that the $\mathrm{MgO}$ thin film shifts the $\operatorname{Ag}(001)$ work function and thus the contact potential by about $1.1 \mathrm{eV}$. This $\mathrm{MgO}$ level is set as the reference level and relative shifts are related to it. From measurements of numerous defects four different types were distinguished by their contact potential, which corresponds to the maximum position of the frequency shift vs bias voltage parabola. The results are shown in Figure 9. On the left-hand

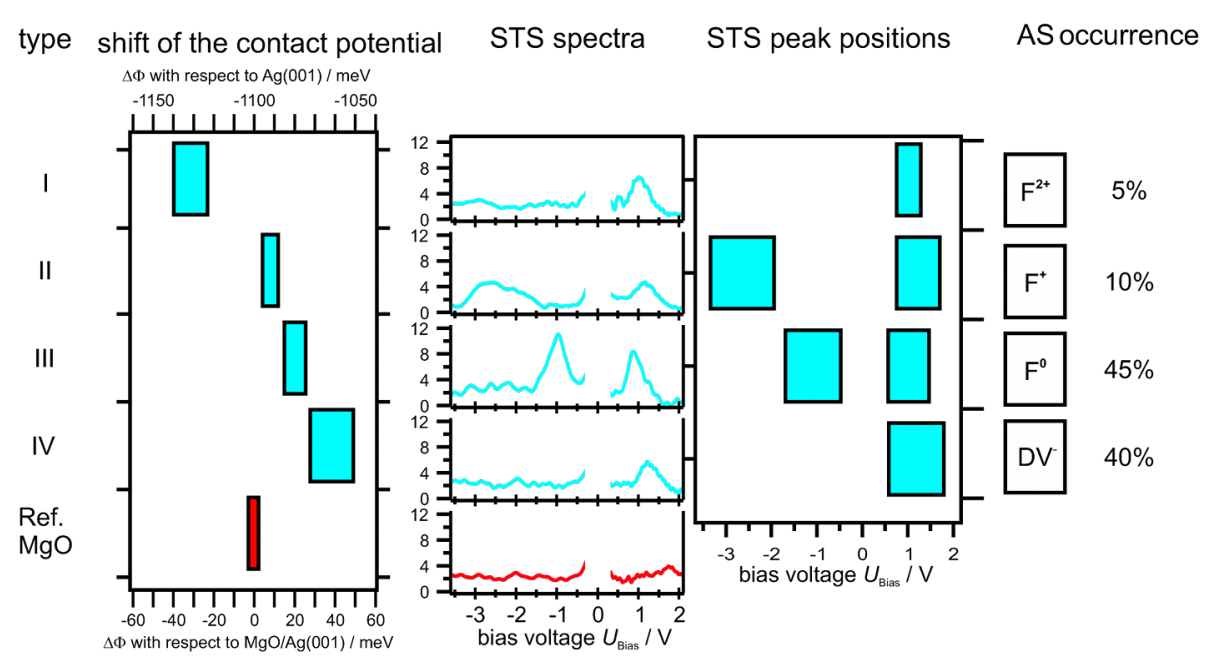

Figure 9: Color centers on MgO. The left labeling assigns numbers to the defect types. The left graph shows the relative shift of the local (effective) contact potential with respect to the $\mathrm{MgO}$ surface (bottom abscissa) and with respect to the $\mathrm{Ag}(001)$ level (top abscissa). The covered range in the shifts results from measurements with different local resolutions due to different tip structures. The energy level scheme presents the different energy levels of the defect types and their local contact potential shifts. The central graph shows STS spectra of the respective defects. The right graph presents the maxima of the STS data. The covered abscissa range accounts for the statistics of the peak positions. The assignment (AS) of defect types to color centers and negatively charged divacancies $\left(\mathrm{DV}^{-}\right)$according to theory as well as their relative occurrence are given on the right hand side. 
side of Figure 9 the four types are indicated by numbers and the $\mathrm{MgO}$ reference level is given (red bar). The graph on the lefthand side represents the measured contact potential with respect to the reference $\mathrm{MgO}$ level (bottom abscissa) and with respect to the $\mathrm{Ag}(001)$ level (top abscissa).

For type I defects shifts of -50 to $-25 \mathrm{meV}$ below the $\mathrm{MgO}$ level were observed. These significant shifts can be explained by the presence of positively charged defects with respect to the surrounding area resulting in a decrease of the local contact potential. The charge density distribution is significantly reduced at the positions of the defects compared with the surrounding $\mathrm{MgO}$ lattice. The presence of charges localized at defect sites induces a contact potential shift of the $\mathrm{MgO} /$ $\operatorname{Ag}(001)$ in analogy to the Helmholtz equation $\Delta \Phi=4 \pi e \mu \sigma$ [37]. Where $\mu$ is the dipole moment induced by the localized charge at the site of the defect and the screening charge in the $\operatorname{Ag}(001)$ substrate and $\sigma$ is the surface concentration. However, the full complexity is not covered by the Helmholtz equation and detailed calculations are still desired. Defect type II shows a contact potential shift of $\approx+9 \mathrm{meV}$. This shift can be assigned to an $\mathrm{F}^{+}$. For an $\mathrm{F}^{+}$the overall charge is positive, but on a very local scale the single electron has a probability above the surface as derived by density functional theory calculations [31]. The charge density spills out of the defect's site and has therefore a probability above the surface. The spill out of the negative charge changes the local dipole moment such that the local contact potential increases compared with the $\mathrm{MgO}$ / $\operatorname{Ag}(001)$ reference level. The electron charge is symmetrically distributed along the surface normal with its charge maximum located in the center of the defect. Defect type III results in a shift of about +15 to $+20 \mathrm{meV}$ above the $\mathrm{MgO}$ level. The shift results from two charges present in a defect site and is thus attributed to an $\mathrm{F}^{0}$ color center. An $\mathrm{F}^{0}$ is neutral compared to the surrounding $\mathrm{MgO}$ lattice, but the two electrons have a large probability density above the surface due to Coulomb repulsion. The charges are as for type II symmetrically distributed and located in the center of the defect, see Figure 8c. Therefore, the charge does not belong to any $\mathrm{Mg}^{2+}$ site surrounding the defect. Thus, the oxidation state of the surrounding lattice is not affected by the trapped charges. The spill out of the charges results in a stronger dipole moment compared to defect type II and the measured shift is about twice as large as that for defect type II.

The strongest positive shift on the relative scale is that of type IV. The strong shift indicates that negative charges are involved. Therefore, this shift might result from divacancies (DV) or $\mathrm{OH}$ groups trapped at low coordinated $\mathrm{Mg}^{2+}$ sites. It is known that $\mathrm{OH}$ groups can trap electrons [38]. However, $\mathrm{OH}$ groups and other adsorbates can be excluded since all defects occur only after high voltage and high current scanning and are not present on regular terraces and steps. With the above mentioned scan parameters, adsorbates would be removed from the scan area. Furthermore, the defects occur only within the high current scan frame and not outside. Favored candidates are, therefore, divacancies formed at step and corner sites since the formation energy at these sites is the lowest. The stability of divacancies and their electron affinity have been confirmed by DFT calculations [39]. A divacancy is neutral compared with the surrounding $\mathrm{MgO}$, since a complete $\mathrm{Mg}-\mathrm{O}$ unit is missing. Due to the electron affinity of $0.6-1 \mathrm{eV}$, electrons can be trapped by the DV from the tunneling junction and the DV becomes negatively charged. The trapped electron of the $\mathrm{DV}^{-}$is strongly localized at the $\mathrm{Mg}^{2+}$ site due to the attractive Coulomb interaction. Since the $\mathrm{DV}^{-}$is negatively charged with respect to the surrounding $\mathrm{MgO}$ area, the additional dipole moment will increase the work function resulting in the largest positive shift on the relative scale. The covered ranges in the maximum positions originate from different tip structures, however, the reproducibility for two subsequent measurements with the same microscopic tip is within $\pm 2 \mathrm{meV}$. All defect types analyzed show a characteristic fingerprint due to different charge states.

The measurements based on NC-AFM are supported by complementary STS. For all defects the local density of states (LDOS) has been detected. The tunneling spectra measurements have been performed directly after the local contact potential measurements without moving the tip laterally, i.e., STS and KPFM have been performed with the same microscopic tip configuration. To prevent tip changes when carrying out STS at high voltages, the feedback on the tunneling current was switched on and $\mathrm{d} z / \mathrm{d} U_{\text {bias }}$ was detected. The $\mathrm{d} z / \mathrm{d} U_{\text {bias }}$ vs $U_{\text {bias }}$ spectrum at constant tunneling current $I_{\mathrm{t}}$ is similar to the $\mathrm{d} I_{\mathrm{t}} / \mathrm{d} U_{\text {bias }}$ vs $U_{\text {bias }}$ spectrum at constant height $z$, see [40].

The tunneling spectra measured on the defects are compared with $\mathrm{MgO}$ spectra on the terrace next to the defect. The $\mathrm{MgO}$ reference spectra show no peaks within the voltage regime due to the band gap (compare red lines in Figure 9). The spectra taken on the $\mathrm{F}^{2+}$ only show peaks in the unoccupied regime at voltages of $\approx+1 \mathrm{~V}$ above the Fermi level (see Figure 9). The $\mathrm{F}^{+}$ centers have both occupied and unoccupied electronic states within the band gap. The electronic states are located within the band gap of $\mathrm{MgO}$. The occupied states are quite broadly distributed from $-3.5 \mathrm{~V}$ to $-2.0 \mathrm{~V}$ below the Fermi level, depending on the defect location on the film [32]. The empty states are at $\approx+1 \mathrm{~V}$ above the Fermi level. Considering the $\mathrm{F}^{0}$ color center, the doubly occupied state is higher in energy, approximately $-1 \mathrm{~V}$ below the Fermi level, while the position of the unoccupied state is similar to $\mathrm{F}^{+}$centers. 
The negatively charged divacancies only show a clear feature in the empty states at about $+1 \mathrm{~V}$. The corresponding occupied shallow state is expected to be very close to the Fermi level, i.e., in a region where the experiment cannot clearly detect states. However, $\mathrm{F}^{0}$ and $\mathrm{DV}^{-}$are equally frequent and represent $\approx 85 \%$ of the total defects. $\mathrm{F}^{+}$color centers are much less frequent and represent $\approx 10 \%$ and $\mathrm{F}^{2+}$ centers about $5 \%$. These findings are in good agreement with the high formation energies of $\mathrm{F}^{2+}$ centers. By comparing the STS peak positions in Figure 9, it becomes obvious that $\mathrm{F}^{2+}$ and $\mathrm{DV}^{-}$defects are hardly distinguishable by their electronic structure but show a significant difference in the local contact potential due to the effect of a locally trapped charge on the surface dipole. This demonstrates the great benefit of NC-AFM and KPFM in combination with STM and STS.

\section{Line defects}

Apart from point defects more complex structures like line defects are found on oxide surfaces. Line defects can be caused by step edges or grain boundaries that penetrate the surface. In thin oxide films line defects are often generated by domain boundaries. The structure at these line defects usually differs significantly from the defect-free domains. This is often associated with a change of electronic properties, which may significantly influence the surface chemistry.

\section{Sample system: aluminum oxide on $\mathrm{NiAl}(110)$}

Thin film aluminum oxide on $\mathrm{NiAl}(110)$ is composed of two oxygen and two aluminum layers limiting the film thickness to $0.5 \mathrm{~nm}$ [41]. It is prepared in a reliable and simple two step oxidation procedure. After dosing $5 \times 10^{-4} \mathrm{~Pa}$ oxygen at $550 \mathrm{~K}$ for
10 minutes, the sample is heated to $1050 \mathrm{~K}$ in vacuum to crystallize the oxide film. This process may be repeated to close open metal patches in the film. The preparation is explained in detail in [42]. The film grows in two reflection domains, A and B. The long edges of the parallelogram shaped unit cells $\left(1.055 \mathrm{~nm} \times 1.788 \mathrm{~nm}, \alpha=88.7^{\circ}\right)$ are rotated by $\pm 24^{\circ}$ with respect to $\mathrm{NiAl}[1 \overline{1} 0]$.

\section{Antiphase domain boundaries in aluminum oxide}

The most common structural defects in the thin film aluminum oxide on $\mathrm{NiAl}(110)$, besides substrate induced step edges, are reflection domain boundaries (from domain $A$ to $B$ or vice versa) and antiphase domain boundaries (abbrev. APDBs; A-A or B-B). The latter are translation domain boundaries originating from strain relief and introduced into already existing oxide patches. For this film system their denotation as APDBs is common usage due to historical reasons and to distinguish them from boundaries between nucleation related translation domains. While the reflection domain boundaries occur less frequent, APDBs occur regularly, approximately every 8-10 nm to release stress in the aluminum oxide film that accumulates due to a small lattice mismatch with the $\mathrm{NiAl}(110)$ surface along the $[1 \overline{1} 0]$ direction.

Different types of APDBs exist, the most common types are straight (type I) and zigzagged (type II) APDBs $[43,44]$. At straight APDBs the surface unit cell is extended parallel to the long edge of the aluminum oxide unit cell. At zigzagged APDBs both directions of the oxide unit cell are extended. For the sake of simplicity, we focus on straight APDBs in this section. A more comprehensive NC-AFM study of the ADPBs

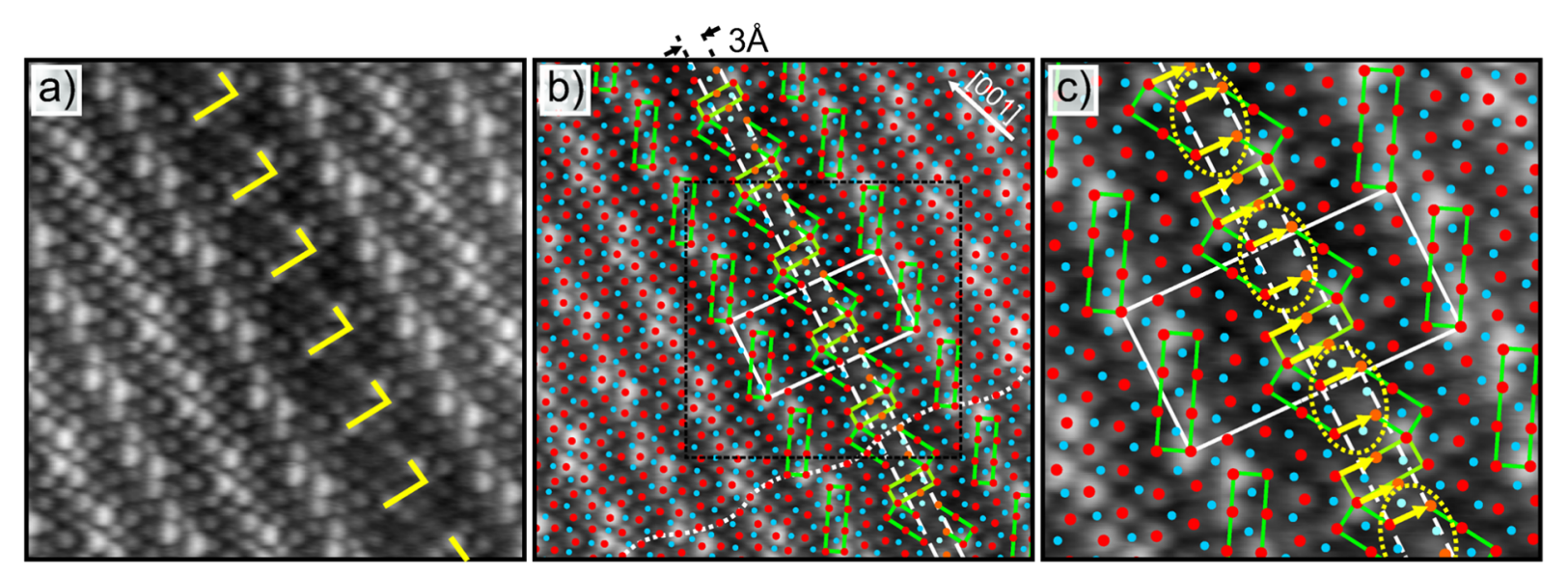

Figure 10: Atomic resolution NC-AFM image of a straight antiphase domain boundary (type I) in the aluminum oxide on NiAl(110). The scan area is $6.4 \mathrm{~nm} \times 6.4 \mathrm{~nm}$ in (a) and (b). b) An adjusted model [47] has been superimposed. The unit cell is extended by $3 \AA$ along the long edge of the unit cell. Inserted sites are given in lighter colors. Dashed lines indicate the extension. The dotted line highlights wave-like oxygen rows along the unit cell. c) shows an enlarged section of the image for better visibility $(3.5 \mathrm{~nm} \times 3.5 \mathrm{~nm})$. Yellow arrows denote the direction and length ( $3 \AA$ ) of the Burgers vector. Yellow loops indicate spacious arrangements of oxygen sites that are different from all domain sites. $\Delta f=-2.75 \mathrm{~Hz}, A_{\text {osc }}=3.8 \AA, U_{\text {bias }}=-220$ $\mathrm{mV}$. 

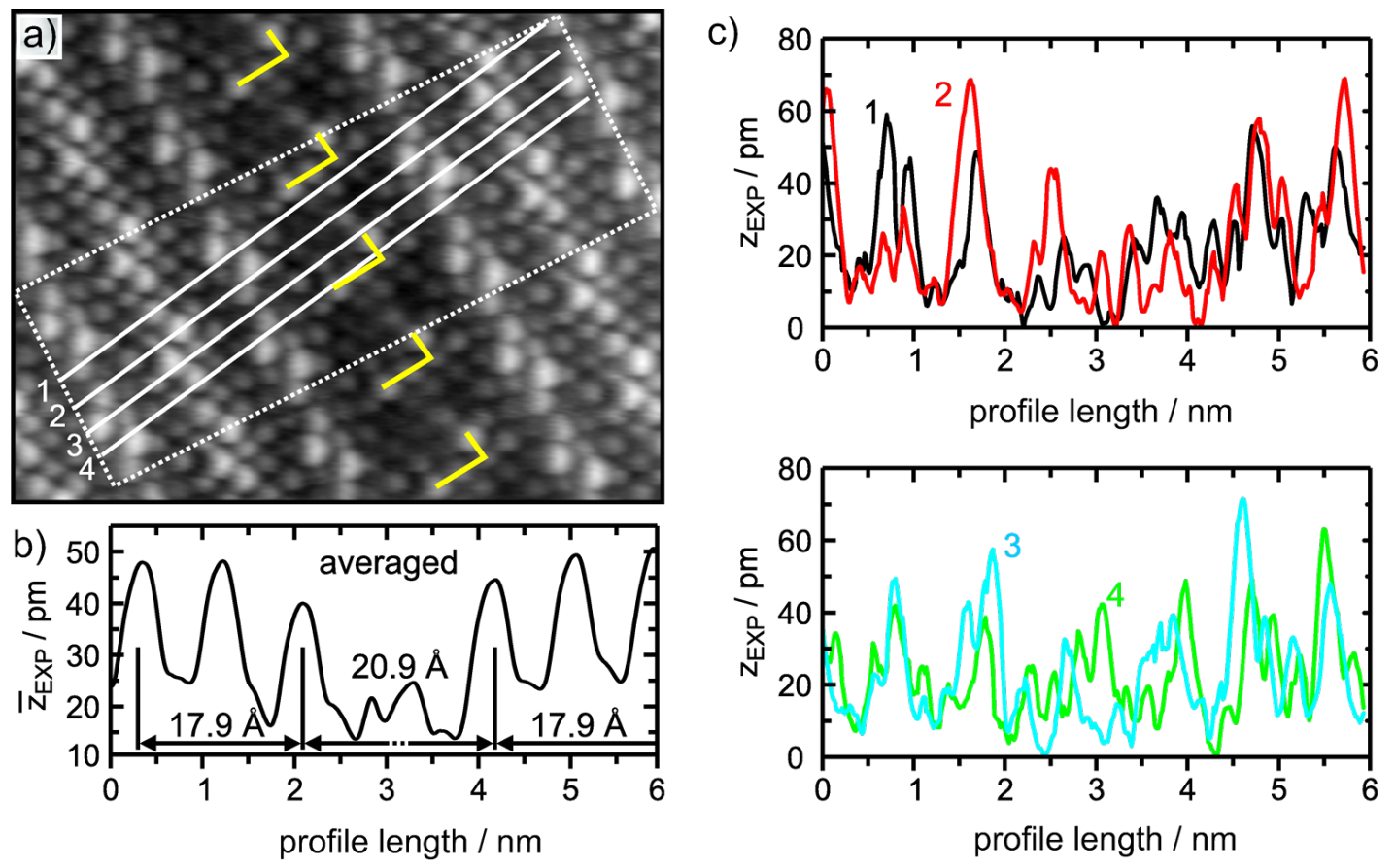

Figure 11: Height profiles. a) Cutout from Figure 10. White lines indicate positions where line profiles have been taken across the type I boundary. b) Averaged line profile taken within the rectangle (two unit cells in width) in the frame above. This emphasizes that such boundaries are reproduced as depressions within NC-AFM images. Averaging was performed over 167 line profiles. c) Single profiles along chains of O atoms across the APDB marked by white lines in the rectangle in (a). Such atom rows show heights different from average terrace height. Only the profile labeled 4 shows nearly no decrease in height over the APDB.

and other line defects on aluminum oxide in $\mathrm{NiAl}(110)$ can be found in $[43,45,46]$. By DFT calculations [47], the stoichiometry of the film with a straight APDB was determined to be $(\mathrm{NiAl})^{2-}$ substrate $\left(\mathrm{Al}_{19} \mathrm{O}_{28} \mathrm{Al}_{28} \mathrm{O}_{32}\right)^{2+}$. An oxygen deficiency with unoccupied electronic states in the aluminum oxide band gap was proposed.

An atomically resolved NC-AFM image of a straight APDB (type B I) is shown in Figure 10. Clearly visible, the boundary is marked by a fairly wide linear depression. The adjusted model for the lateral positions at the APDB [47] is superimposed in Figure 10b and found to be in perfect agreement. From this we see that NC-AFM images the surface oxygen sites of the film with high accuracy. The model is based on a unit cell that has been split in the middle according to STM images. Important structural elements of the oxygen sub-lattice are highlighted as well as the extended unit cell and two equivalent lines between which the inserted new sites are visible. Inserted sites are marked in a slightly different color to distinguish them from the usual sites in the oxide unit cell: orange and light blue as compared to red and blue. In Figure 10c an enlarged section of the elongated unit cell at the APDB is given. In the middle of the APDB a broken block of $8 \mathrm{O}$ atoms appears, which is of the type that is almost aligned with the NiAl[001] direction. A particularly spacious arrangement of oxygen atoms in the shape of a quadrangle (yellow dotted loops) is formed at this block at the boundary. This is in agreement with DFT calculations [47], which assign an electronic defect state to this structure. Another deviation from the usual oxide unit cell is a rectangle of six oxygen sites which is derived from the bridging square groups indicated in light green in Figure 10c. These characteristic protrusions in the topography of the boundary form the shape of the letter ' $L$ ' as indicated by the yellow angle in Figure 10a.

The direction and the length of the lattice discrepancy generated by a dislocation in a crystal is given by the Burgers vector. At straight APDBs this vector measures $3 \AA$ in length and is parallel to the long edge of the oxide unit cell as indicated by yellow arrows. At the same time the Burgers vector is also parallel to the overall direction of the wave-like rows of atoms within the surface aluminum and oxygen sub-lattices (dotted line in Figure 10b). Considering the topographic quality of the contrast, the domain boundary can finally be determined to be a depression. This is summarized in Figure 11. An averaged line profile across the APDB I covering the width of 2 unit cells, as indicated by the rectangle in Figure $11 \mathrm{a}$, is shown in Figure 11b. In Figure 11c individual line profiles across oxygen rows are shown. These profiles have been taken along the white 

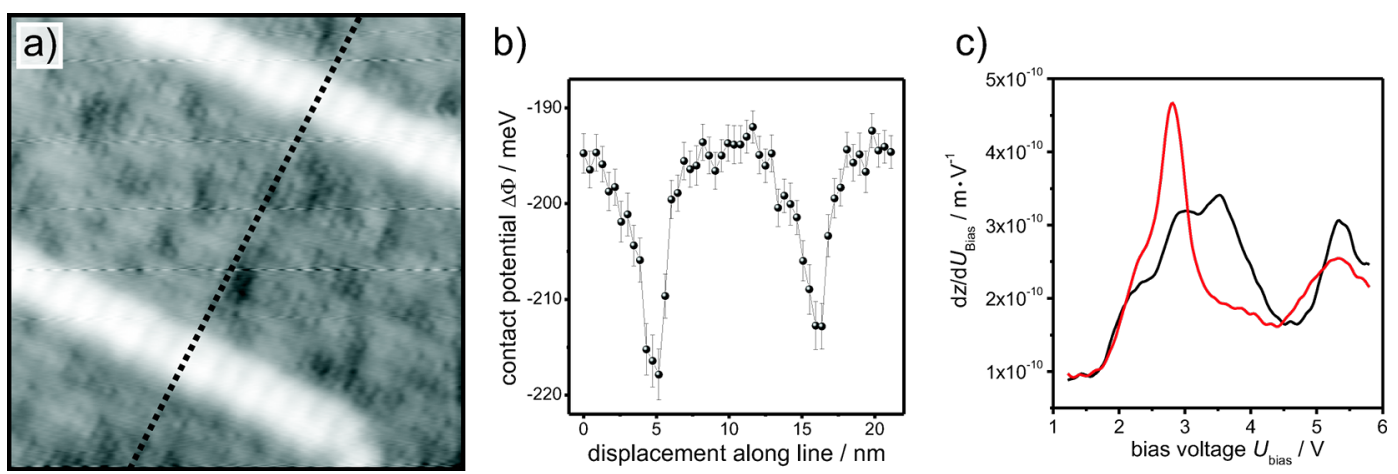

Figure 12: Spectroscopy on aluminum oxide. a) STM image of a thin film of aluminum oxide on $\operatorname{NiAl}(110), 18 \mathrm{~nm} \times 18 \mathrm{~nm}$. Two straight APDBs (bright) separating three $A$ domains (dark) are visible. $U_{\text {bias }}=+3 \mathrm{~V}$ and $I_{t}=100 \mathrm{pA}$. b) Effective contact potential, which was determined at the line shown in (a), decreases at the APDB by approximately $20 \mathrm{meV}$. The tip height was constant during the measurement and corresponds to a frequency shift of $-1 \mathrm{~Hz}$ at $-150 \mathrm{mV}$. The contact potentials were determined by fitting the frequency shift vs bias voltage curves with a quadratic term (see Equation 5). The error bars represent the accuracies by which the maxima were determined. c) STS curves taken at the domain (black) and the APDB (red).

lines 1, 2, 3, 4 in the image in (a). At the linear oxygen rows there exist sites with a mean height that is $10 \mathrm{pm}$ lower than the average height of corresponding terrace sites (see Figure 11b).

Knowing the surface structure with highest accuracy, it is still very desirable to determine aspects of electronic structures to gain further insight. In Figure 12, the effective contact potential is plotted for positions along a line across three domains and two straight APDBs. The recorded contact potential at the APDB is approximately $20 \mathrm{meV}$ smaller than at the regular domain, which was verified at many different sites [45]. This means the work function at the APDBs is reduced compared to that of the domain. Comparison of STS curves on domain and APDB shows significant differences in the electronic structures. At the domain boundary a pronounced unoccupied defect state appears at bias voltages between $2-3 \mathrm{~V}$.

The real variation of contact potential may be even larger, since the recorded signal is a convolution of the actual contact potential difference with the tip geometry [48]. The depressions in the contact potential at the APDBs have approximately a full width at half minimum of $3 \mathrm{~nm}$ (see Figure 12b). The lateral extension of the APDBs is approximately $1.5 \mathrm{~nm}$ and the oxide unit cell is expanded by an additional row of oxygen atoms by $0.3 \mathrm{~nm}$ at that position [41]. Assuming the change of the contact potential to be approximately located in this range (between 0.3 and $1.5 \mathrm{~nm}$ ), the recorded contact potential is broadened by a factor of 2 to 10 due to the convolution with the tip geometry. This means on the other hand, the contact potential difference is actually 2 to 10 times larger than recorded. Furthermore, we conclude that the tip is influenced by these defects over a distance of approximately $2-3 \mathrm{~nm}$, which might be a reasonable estimation of the tip size. This gives also the lateral resolution of the measurements. As shown in [36] and [49], the determined contact potential difference depends also on the tipsample distance. In general, a smaller distance increases the size of the interaction and decreases the integration area, this means the determined difference of the contact potential increases. If the distance is too small, the probability that the tip restructures increases. Therefore, the tip-sample distance was set to a moderate value which corresponds to roughly $0.5-0.75$ of the maximum absolute frequency shift.

Thin film aluminum oxide reduces the work function on $\mathrm{NiAl}(110)$ by approximately $0.5 \mathrm{eV}$ from $4.8 \mathrm{eV}$ for a pure $\mathrm{NiAl}(110)$ surface to $4.3 \mathrm{eV}$ for the aluminum oxide film [50]. A further reduction of the work function at the APDBs may explain the higher reactivity at these linear defects. In [51], it has been shown that APDBs are preferred adsorption sites for different atoms and metal clusters. Furthermore, a particular chemical activity at the APDBs has been experimentally verified. For instance, nitric oxide decomposition on thin film aluminum oxide preferentially takes place at the APDBs [52].

It has been predicted by means of DFT calculations [47] that unoccupied defect states in the APDBs of the aluminum oxide film are associated with $\mathrm{F}^{2+}$-like centers. In our NC-AFM measurements, we have recorded a shift of the local work function of approximately $-20 \mathrm{meV}$ at the APDB. This is in great agreement with the shift recorded at $\mathrm{F}^{2+}$ centers on $\mathrm{MgO}$ / $\mathrm{Ag}(001)$ (see Figure 9). As it has been shown above, a small shift of the contact potential difference is caused by the fact that the recorded contact potential depends on the tip-sample distance. The recorded change of the work function is in agreement with the DFT calculations, where a shift of the valence and the conduction band with a local band bending at the APDB 
were predicted [47]. Thus, in the APDB $\mathrm{F}^{2+}$-like centers, which have been predicted by DFT calculations, are now experimentally verified by NC-AFM.

\section{Conclusion}

Defects on surfaces of thin oxide films were studied by means of low temperature NC-AFM combined with STM in UHV. In addition to imaging the topography of the surface termination, STS and KPFM were employed for a deeper insight into the nature of the defects. The spectroscopy was performed with a very high spatial resolution in the order of $1 \mathrm{~nm}$. For magnesium oxide on $\operatorname{Ag}(001)$, different point defects, which are the most frequently discussed ones in literature, were studied. Using contact potential measurements by KPFM in comparison to STS spectra and DFT calculations, the point defects on an $\mathrm{MgO}$ surface could be unambiguously identified for the first time. The point defects were distinguished as $\mathrm{DV}^{-}, \mathrm{F}^{0}, \mathrm{~F}^{+}$and $\mathrm{F}^{2+}$ color centers. In addition, the electronic signature was measured and electronic defect states were determined within the band gap of the $\mathrm{MgO}$ surface. These color centers influence the surface chemistry by significantly increasing the reactivity of the almost inert surface of defect-free MgO. The NC-AFM investigation on aluminum oxide on $\mathrm{NiAl}(110)$ unveils the surface structure of the domain and at the APDBs with atomic resolution. Apart from the determined topography, $\mathrm{F}^{2+}$-like centers, which have been predicted by DFT calculations, were experimentally verified for the APDBs. These studies show that NC-AFM in combination with STM can be successfully used beyond imaging the topography of the surface termination. The employed high resolution spectroscopy significantly improves our understanding of the surface chemistry of thin oxide films.

\section{Acknowledgements}

Hans-Joachim Freund is gratefully acknowledged for his help, support and advise. Many fruitful and illuminating discussions with Gianfranco Pacchioni are also gratefully acknowledged. The authors would like to thank Hans-Peter Rust and Gero Thielsch for their great technical contributions and Christin Büchner for fruitful discussions.

\section{References}

1. Ertl, G.; Knözinger, H.; Schüth, F.; Weitkamp, J., Eds. Handbook of heterogeneous catalysis, 2nd ed.; Wiley-VCH: Weinheim, Germany, 2008; Vol. 2.

2. Freund, H.-J. Chem.-Eur. J. 2010, 16, 9384-9397. doi:10.1002/chem.201001724

3. Plummer, E.; Matzdorf, R.; Melechko, A.; Zhang, J. Prog. Surf. Sci. 2001, 67, 17-44. doi:10.1016/S0079-6816(01)00014-4

4. Henrich, V. E.; Cox, P. A. The surface science of metal oxides; Cambridge University Press: Cambridge, U. K., 1996.

5. Nilius, N. Surf. Sci. Rep. 2009, 64, 595-659. doi:10.1016/j.surfrep.2009.07.004
6. Tilley, R. J. D. Defect crystal chemistry and its applications; Chapman and Hall: New York, U. S. A., 1987.

7. König, T.; Simon, G. H.; Rust, H.-P.; Heyde, M. Appl. Phys. Lett. 2009, 95, 083116. doi:10.1063/1.3189282

8. Heyde, M.; Simon, G. H.; Rust, H.-P.; Freund, H.-J. Appl. Phys. Lett. 2006, 89, 263107. doi:10.1063/1.2424432

9. Israelachvili, J. Intermolecular \& Surface Forces; Academic Press: Burlington, MA, U. S. A., 1991.

10. Chen, C. J. Introduction to scanning tunneling microscopy; Oxford University Press: Oxford, U. K., 2008.

11. London, F. Z. Phys. 1930, 63, 245-279. doi:10.1007/BF01421741

12. Sader, J. E.; Jarvis, S. P. Appl. Phys. Lett. 2004, 84, 1801-1803. doi:10.1063/1.1667267

13. Nonnenmacher, M.; Oboyle, M.; Wickramasighe, H. Appl. Phys. Lett. 1991, 58, 2921-2923. doi:10.1063/1.105227

14. Jacobs, H.; Leuchtmann, P.; Homan, O.; Stemmer, A. J. Appl. Phys. 1998, 84, 1168-1173. doi:10.1063/1.368181

15. Filleter, T.; Emtsev, K. V.; Seyller, T.; Bennewitz, R. Appl. Phys. Lett. 2008, 93, 133117. doi:10.1063/1.2993341

16. Kawai, S.; Glatzel, T.; Hug, H.-J.; Meyer, E. Nanotechnology 2010, 21, 245704. doi:10.1088/0957-4484/21/24/245704

17. Kelvin, W. T. L. Philos. Mag. 1898, 46, 82-120.

18. Stomp, R.; Miyahara, Y.; Schaer, S.; Sun, Q.; Guo, H.; Grütter, P.; Studenikin, S.; Poole, P.; Sachrajda, A. Phys. Rev. Lett. 2005, 94, 056802. doi:10.1103/PhysRevLett.94.056802

19. Waser, R., Ed. Nanoelectronics and Information Technology, 2nd ed.; Wiley-VCH: Weinheim, Germany, 2005; pp 426-427.

20. Yang, K.-M.; Chung, J.-Y.; Hsieh, M.-F.; Lin, D.-S Jpn. J. Appl. Phys., Part 1 2007, 46, 4395-4402. doi:10.1143/JJAP.46.4395

21. Palermo, V.; Palma, M.; Samorì, P. Adv. Mater. 2006, 18, 145-164. doi:10.1002/adma.200501394

22. Pacchioni, G. Theory of point defects at the MgO surface. In Oxide surfaces; Woodruff, D. P., Ed.; Chemical Physics Of Solid Surfaces, Vol. 9; Elsevier: Amsterdam, 2001; pp 94-135. doi:10.1016/S1571-0785(01)80023-7

23. Ferrari, A. M.; Pacchioni, G. J. Phys. Chem. 1995, 99, 17010-17018. doi:10.1021/j100046a029

24. Scorza, E.; Birkenheuer, U.; Pisani, C. J. Chem. Phys. 1997, 107, 9645-9658. doi:10.1063/1.475260

25. Barth, C.; Henry, C. R. Phys. Rev. Lett. 2003, 91, 196102. doi:10.1103/PhysRevLett.91.196102

26. Livshits, A. L.; Shluger, A. L.; Rohl, A. L.; Foster, A. S. Phys. Rev. B: Condens. Matter Mater. Phys. 1999, 59, 2436-2448. doi:10.1103/PhysRevB.59.2436

27. Sushko, P. V.; Shluger, A. L.; Catlow, C. R. A. Surf. Sci. 2000, 450, 153-170. doi:10.1016/S0039-6028(00)00290-9

28. Corneille, J. S.; He, J.-W.; Goodman, D. W. Surf. Sci. 1994, 306, 269-278. doi:10.1016/0039-6028(94)90071-X

29. Fang, C. S. A. Surf. Sci. 1990, 235, L291-L294. doi:10.1016/0039-6028(90)90098-S

30. Trevethan, T.; Shluger, A. Nanotechnology 2009, 20, 264019. doi:10.1088/0957-4484/20/26/264019

31. Giordano, L.; Martinez, U.; Pacchioni, G.; Watkins, M.; Shluger, A. L. J. Phys. Chem. C 2008, 112, 3857-3865. doi:10.1021/jp7108016

32. Sterrer, M.; Heyde, M.; Novicki, M.; Nilius, N.; Risse, T.; Rust, H.; Pacchioni, G.; Freund, H. J. J. Phys. Chem. B 2006, 110, 46-49. doi:10.1021/jp056306f

33. Sicolo, S.; Giordano, L.; Pacchioni, G. J. Phys. Chem. C 2009, 113, 16694-16701. doi:10.1021/jp905592c 
34. Giordano, L.; Pacchioni, G. Phys. Chem. Chem. Phys. 2006, 8, 3335-3341. doi:10.1039/b604288k

35. König, T.; Simon, G. H.; Rust, H.-P.; Pacchioni, G.; Heyde, M.; Freund, H.-J. J. Am. Chem. Soc. 2009, 131, 17544-17545. doi:10.1021/ja908049n

36. König, T.; Simon, G. H.; Rust, H.-P.; Heyde, M. J. Phys. Chem. C 2009, 113, 11301-11305. doi:10.1021/jp901226q

37. Somorjai, G. A. Introduction to Surface Chemistry and Catalysis; John Wiley \& Sons, Inc.: New York, 1994.

38. Napoli, F.; Chiesa, M.; Giamello, E.; Finazzi, E.; Di Valentin, C.; Pacchioni, G. J. Am. Chem. Soc. 2007, 129, 10575-10581. doi:10.1021/ja073114k

39. Ricci, D.; Pacchioni, G.; Sushko, P. V.; Shluger, A. L. J. Chem. Phys. 2002, 117, 2844-2851. doi:10.1063/1.1491405

40. Ziegler, M.; Néel, N.; Sperl, A.; Kröger, J.; Berndt, R. Phys. Rev. B 2009, 80, 125402. doi:10.1103/PhysRevB.80.125402

41. Kresse, G.; Schmid, M.; Napetschnig, E.; Shishkin, M.; Köhler, L.; Varga, P. Science 2005, 308, 1440-1442. doi:10.1126/science.1107783

42. Jaeger, R.; Kuhlenbeck, H.; Freund, H.-J.; Wuttig, M.; Hoffmann, W.; Franchy, R.; Ibach, H. Surf. Sci. 1991, 259, 235-252. doi:10.1016/0039-6028(91)90555-7

43. Simon, G. H.; König, T.; Rust, H.-P.; Heyde, M.; Freund, H.-J. New J. Phys. 2009, 11, 093009. doi:10.1088/1367-2630/11/9/093009

44. Kulawik, M.; Nilius, N.; Rust, H. P.; Freund, H. J. Phys. Rev. Lett. 2003, 91, 256101. doi:10.1103/PhysRevLett.91.256101

45. Heinke, L.; Lichtenstein, L.; Simon, G. H.; König, T.; Heyde, M.; Freund, H.-J. Phys. Rev. B 2010, 82, 075430. doi:10.1103/PhysRevB.82.075430

46. Heinke, L.; Lichtenstein, L.; Simon, G.; König, T.; Heyde, M.; Freun, H.-J. ChemPhysChem 2010, 11, 2085-2087. doi:10.1002/cphc.201000060

47. Schmid, M.; Shishkin, M.; Kresse, G.; Napetschnig, E.; Varga, P.; Kulawik, M.; Nilius, N.; Rust, H. P.; Freund, H. J. Phys. Rev. Lett. 2006, 97, 046101. doi:10.1103/PhysRevLett.97.046101

48. Zerweck, U.; Loppacher, C.; Otto, T.; Grafstrom, S.; Eng, L. Phys. Rev. B 2005, 71, 125424. doi:10.1103/PhysRevB.71.125424

49. Gross, L.; Mohn, F.; Liljeroth, P.; Repp, J.; Giessibl, F. J.; Meyer, G. Science 2009, 324, 1428-1431. doi:10.1126/science.1172273

50. Song, W.; Yoshitake, M. Appl. Surf. Sci. 2005, 251, 14-18. doi:10.1016/j.apsusc.2005.03.116

51. Bäumer, M.; Freund, H.-J. Prog. Surf. Sci. 1999, 61, 127-198. doi:10.1016/S0079-6816(99)00012-X

52. Schauermann, S.; Johánek, V.; Laurin, M.; Libuda, J.; Freund, H.-J. Chem. Phys. Lett. 2003, 381, 298. doi:10.1016/j.cplett.2003.09.101

\section{License and Terms}

This is an Open Access article under the terms of the Creative Commons Attribution License

(http://creativecommons.org/licenses/by/2.0), which permits unrestricted use, distribution, and reproduction in any medium, provided the original work is properly cited.

The license is subject to the Beilstein Journal of Nanotechnology terms and conditions:

(http://www.beilstein-journals.org/bjnano)

The definitive version of this article is the electronic one which can be found at:

doi:10.3762/bjnano.2.1 